\title{
Retreatment with lenalidomide is an effective option in heavily pretreated refractory multiple myeloma patients
}

\author{
M. STORK ${ }^{1}$, S. SEVCIKOVA ${ }^{2}$, Z. ADAM ${ }^{1}$, M. KREJCI ${ }^{1}$, V. SANDECKA ${ }^{1}$, Z. KRAL ${ }^{1}$, L. BROZOVA ${ }^{3}$, R. VELICHOVA ${ }^{3}$, L. POUR ${ }^{1, *}$ \\ ${ }^{1}$ Department of Hematology and Oncology, University Hospital Brno, Brno, Czech Republic; ${ }^{2}$ Babak Myeloma Group, Department of Pathologi- \\ cal Physiology, Faculty of Medicine, Masaryk University, Brno, Czech Republic; ${ }^{3}$ Institute of Biostatistics and Analyses, Faculty of Medicine, \\ Masaryk University, Brno, Czech Republic
}

${ }^{*}$ Correspondence: pour.ludek@fnbrno.cz

Received May 19, 2017 / Accepted September 27, 2017

\begin{abstract}
The combination of lenalidomide and dexamethasone is the current gold standard for treatment of relapsed multiple myeloma. This study analyzes the efficiency of repeated lenalidomide treatment in patients with relapsed and refractory multiple myeloma. A total of 41 patients were prospectively evaluated at the University Hospital Brno. Lenalidomide was administered at standard dosing and in combination with corticosteroids and/or chemotherapy. The maximum cumulative dose of lenalidomide was limited to $4,200 \mathrm{mg}$ because of Czech health insurance rules. Before the second lenalidomide treatment, all patients were refractory to the last treatment; previously, $95 \%$ of patients had bortezomib treatment, $48 \%$ had autologous transplantation and the median number of prior therapy lines was three. A partial $14.2 \%$ or better response was achieved with the second lenalidomide treatment. The median progression-free survival was 4.8 months, and median overall survival was 11.9 months. Unfortunately, predicting risk factors in lenalidomide retreatment proved unsuccessful. Although our treatment results were significantly affected by limited Czech health care system coverage for lenalidomide, we established that its repeated treatment is an effective therapeutic alternative for heavily pretreated patients with relapsed and refractory multiple myeloma.
\end{abstract}

Key words: multiple myeloma, lenalidomide, retreatment

Multiple myeloma (MM) is caused by uncontrolled proliferation of malignant plasma cells in the bone marrow, and it is the second most common hematological malignancy. Its prognosis in the era of conventional chemotherapy was not good, with median overall patient survival not longer than 30 months. Introduction of immunomodulatory drugs (lenalidomide, pomalidomide) and the proteasome inhibitors (bortezomib and carfilzomib) provided fundamental improvement in prognosis of newly diagnosed and relapsed MM patients [1]. Although it is generally accepted that relapsed MM is incurable [2], the efficiency of these drugs made it possible that a specific group of MM patients may even be cured. Relapsed and refractory MM patients have poor prognosis and their treatment options are often limited $[3,4]$. Bortezomib-based regimen is a current standard treatment for newly diagnosed MM patients, and its combination with lenalidomide is widely used in the Czech Republic for initial relapse [5-9]. Lenalidomide is an immunomodulatory drug with high effectiveness in both primotherapy and in relapsed MM [7-10]. Retreatment with lenalidomide has not been widely reported, especially in case of refractory patients [11]. This study analyzes the lenalidomide retreatment in relapsed and refractory MM patients.

\section{Patients and methods}

We prospectively analyzed 41 patients repeatedly treated by lenalidomide-containing regimen. All patients were eligible for this treatment. Before the second lenalidomide treatment, other option was only palliative care. The patients were treated at the Brno University Hospital from June 2009 to December 2015, and all signed informed consent before entering this study. The study was approved by the Hospital Ethics committee in accordance with the current Helsinki Declaration.

The primary endpoint of this analysis was progressionfree survival (PFS) and overall survival (OS) for lenalidomide retreatment. Response was assessed according to the 2014 
International Myeloma Working Group (IMWG) criteria [12]. A total of 22 women and 19 men were included in the study with median age at first-line lenalidomide therapy of 67 years (range 53-78) and 69 years in the second line (range $55-81)$. The median of prior lines of therapy at the time of first-line lenalidomide treatment was 1 (range 0-3) and 3 (range 2-6) at the second-line treatment. Before second-line lenalidomide treatment; (1) 95.1\% (39/41) of patients were pretreated with bortezomib and $61.0 \%(25 / 41)$ with thalidomide. (2) 48.7\% (20/41) underwent high dose chemotherapy followed by peripheral blood stem cell transplant (PBSCT) and (3) all patients were refractory to their last line of treatment. There was no difference in the number of extramedullary disease between the first and second lines of lenalidomide treatment $(12.2 \%$ vs. $14.6 \%, p=1.000)$, nor was there any significant difference in the ISS or DS stage of disease. Table 1 contains further characteristics. Lenalidomide was primarily administered orally at $25 \mathrm{mg}$ /day dosage for 21 days in a 28 -day cycle. This was reduced, as recommended, to $10 \mathrm{mg}$ or $5 \mathrm{mg}$ when necessary [13]. The median number of administered lenalidomide cycles was 8 (range 1-14) in the first lenalidomide treatment, and 4 (range 1-11) in the second treatment. The Czech health insurance system ruled that lenalidomide was not reimbursed if the patient had not achieved at least partial response after 4 cycles. Further, lenalidomide cost was only reimbursed until the total cumulative dose of 4,200 $\mathrm{mg}$ was reached in one line of treatment.

Table 1. Patient demographic and baseline characteristics.

\begin{tabular}{|c|c|c|}
\hline $\begin{array}{l}\text { Basic characteristics } \\
(\mathrm{N}=41)\end{array}$ & $\begin{array}{c}\text { First treatment } \\
\text { with lenalidomide }\end{array}$ & $\begin{array}{l}\text { Second treatment } \\
\text { with lenalidomide }\end{array}$ \\
\hline \multicolumn{3}{|l|}{ Myeloma type, $\mathrm{n}(\%)$} \\
\hline $\operatorname{IgG}$ & \multicolumn{2}{|c|}{$26(63.4)$} \\
\hline IgA & \multicolumn{2}{|c|}{$8(19.5)$} \\
\hline LC only & \multicolumn{2}{|c|}{$4(9.8)$} \\
\hline Nonsecretory & \multicolumn{2}{|c|}{$3(7.3)$} \\
\hline \multicolumn{3}{|l|}{ Durie-Salmon stage, $\mathrm{n}(\%)$} \\
\hline I & $4(9.8)$ & $3(7.3)$ \\
\hline II & $5(12.2)$ & $5(12.2)$ \\
\hline III & $32(78.0)$ & $33(80.5)$ \\
\hline $\begin{array}{l}\text { Durie-Salmon substage } \\
\mathrm{A} / \mathrm{B}, \mathrm{n}(\%)\end{array}$ & $41 / 0(100 / 0)$ & $39 / 2(95.1 / 4.9)$ \\
\hline \multicolumn{3}{|l|}{ ISS stage, $\mathrm{n}(\%) ; \mathbf{N}=\mathbf{4 0}$} \\
\hline Stage 1 & $17(42.5)$ & $13(32.5)$ \\
\hline Stage 2 & $14(35.0)$ & $15(37.5)$ \\
\hline Stage 3 & $9(22.5)$ & $12(30.0)$ \\
\hline \multicolumn{3}{|l|}{ Line of treatment, $\mathrm{n}(\%)$} \\
\hline primotherapy & $5(12.2)$ & $0(0)$ \\
\hline 2nd & $19(46.3)$ & $0(0)$ \\
\hline $3 \mathrm{rd}$ & $12(29.3)$ & $10(24.4)$ \\
\hline 4th and more & $5(12.2)$ & $31(75.6)$ \\
\hline 7th and more & $0(0)$ & $8(19.5)$ \\
\hline
\end{tabular}

Count (relative frequencies) for categorical variables and median $\left(5^{\text {th }}-95^{\text {th }}\right.$ percentiles) for continuous variables
Patients received low molecular weight heparin or aspirin as prophylaxis for thromboembolic complications according to risk stratification [14]. Lenalidomide was most often administered with cyclophosphamide and corticosteroids $(55 \%, 22 / 41)$, or only with corticosteroids $(30 \%, 12 / 41)$ in first-line treatment. In the second-line therapy, lenalidomide was most often administered with corticosteroids $(70.7 \%$, 29/41). The first lenalidomide treatment was followed by PBSCT in 14.6\% (6/41) of patients and by a second treatment with lenalidomide in $9.8 \%$ (4/41) of patients. Adverse events (AEs) were graded as established in the National Cancer Institute Common Terminology Criteria for Adverse Events version 3.0 and safety was evaluated both throughout the study and during long-term follow-up.

Statistical analysis Data was described by absolute and relative frequencies for categorical variables and median supplemented by $5^{\text {th }}-95^{\text {th }}$ percentile range for continuous variables. The differences in basic characteristics for first and second lenalidomide treatments were tested by the McNemar paired test for categorical variables and the Wilcoxon paired signed-rank test for continuous variables. The univariate Cox proportional hazards model evaluating the association of predictors with PFS and PFS and TTP in both first and second treatments were visualised by Kaplan-Meier methodology and compared using the log-rank test. Two-sided tests were used with significance level of 0.05 and analysis was conducted in SPSS software (IBM Corp. Released 2013 IBM SPSS Statistics for Windows, Version 23.0. Armonk, NY: IBM Corp). Finally, figures were plotted using $\mathrm{R}$ version 3.3.0 (www.r-project.org).

\section{Results}

Treatment response. Treatment response could be evaluated in $85 \%$ of patients $(35 / 41)$, and overall response rate (ORR - partial response or better) was achieved by significantly more patients in the first line of lenalidomide treatment than in the second $-68.6 \%(24 / 35)$ vs. $14.2 \%(5 / 35)$, $\mathrm{p}<0.001$.

The following responses were achieved; (1) complete response (CR) and stringent complete response (sCR) was achieved in the first line of treatment in $14.2 \%(5 / 35)$ of patients, but not in the second line; (2) very good partial response (VGPR) occurred significantly more often in the first treatment than in retreatment $-25.7 \%(9 / 35)$ vs. $5.7 \%$ (2/35), $\mathrm{p}=0.039$; (3) partial response (PR) was significantly more often registered in the first line of treatment than in retreatment $-28.6 \%(10 / 35)$ vs. $8.6 \%(3 / 35), \mathrm{p}=0.039$ and $(4)$ minor response (MR) was achieved equally in both lines of treatment $-5.7 \%$ vs. $5.7 \%(2 / 35), \mathrm{p}=1.00$.

Similar number of patients in these lines had stable disease (SD) $-2.9 \%(1 / 35)$ versus $17.1 \%(6 / 35), p=0.125$. In the retreated group, $14.2 \%(5 / 35)$ of patients achieved SD after only the fourth cycle of treatment; and this accounted for $83 \%(5 / 6)$ of all of patients with SD. Treatment in these 
Table 2. Characteristics before second administration of lenalidomide. (patients with final treatment response PR or better in second therapy with lenalidomide; $\mathrm{N}=5$ patients). Previous treatment lines before lenalidomide retreatmetnt

\begin{tabular}{llllll}
\hline Patient No. & $\mathbf{1}^{\text {st }}$ line & $\mathbf{2}^{\text {nd }}$ line & $\mathbf{3}^{\text {rd }}$ line & $\mathbf{4}^{\text {th }}$ line & PBSCT \\
\hline $\mathbf{1}$ & BDD +PBSCT & CTD & RAD & VD & BDD \\
$\mathbf{3}$ & CTD & BDD & RCP & & \\
$\mathbf{4}$ & RD & CVD + PBSCT & BDD & & \\
5 & RP & BDD & & & \\
\hline
\end{tabular}

Abbreviations: BDD- bortezomib-doxorubicin-dexamethasone; PBSCT - peripheral blood stem cell transplant; CTD - cyclophosphamide-thalidomidedexamethasone; RAD - lenalidomide-doxorubicin-dexamethasone; RCP - lenalidomide-cyclophosphamide-prednisone; VD - bortezomib-dexamethasone; RD- lenalidomide- dexamethasone; CVD - cyclophosphamide-bortezomib-dexamethasone; RP- lenalidomide-prednisone; VTP - bortezomibthalidomide-prednisone

patients was stopped according to Czech health insurance rules. Significantly fewer patients progressed during treatment after the first treatment than after retreatment $22.9 \%(8 / 35)$ vs. $62.9 \%(22 / 35) ; \mathrm{p}=0.003$. Only $4.9 \%(2 / 41)$ of patients in the first group and $19.5 \%(8 / 41)$ of patients in the second group progressed after the first 1-2 treatment cycles. Only $36 \%(8 / 22)$ of patients with disease progression progressed in the first two cycles of lenalidomide retreatment. From all patients who progressed after second lenalidomide treatment, $18 \%(4 / 22)$ achieved at least PR before treatment withdrawal. It was the same number of patients $(9.8 \%$ (4/41), who achieved treatment response (PR or better), but progressed after treatment withdrawal. Patients who did not achieve at least $P R$ had a median of 3 treatment cycles in both lines of treatment. Finally, Table 2 lists the previous lines of treatment for patients who achieved treatment response after the second lenalidomide treatment.

Survival intervals. Median PFS was statistically significantly longer in the first lenalidomide treatment at 15.2 months compared to 4.8 months in the second treatment $(\mathrm{p}<0.001)$. Unfortunately, $14.2 \%(5 / 35)$ of patients had to stop the second lenalidomide treatment after 4 cycles due to lenalidomide reimbursement rules, regardless of disease progression. The PFS at 12 months was also significantly longer in the first lenalidomide treatment $(73.2 \%$ vs. $15.1 \%$; $\mathrm{p}<0.001$ ) (Figure 1, Table 3).

Time to progression (TTP) was significantly longer in the first lenalidomide treatment (median 15.2 months vs. 4.8 months, $\mathrm{p}<0.001$ ), and duration of response (DOR) was longer in the first lenalidomide treatment (14.8 months vs. 10.6 months; $\mathrm{p}=0.042$ ). Treatment response lasted in $66.7 \%$ of patients at 12 months compared to $20.5 \%$ without response, and the median OS in repeated lenalidomide treatment was 11.9 months [95\% CI: 10.1-13.7].

Subgroup analysis. No risk factor predicting successful repeated lenalidomide treatment was found. Dependency on previous lenalidomide treatment response was not proven; HR 1.043 (95\% CI: 0.495-2.200; $\mathrm{p}=0.912$ ). Furthermore, no dependency was determined on the number of previous lines of therapy prior to first and second lenalidomide treatments or the time between lenalidomide treatments. Previous

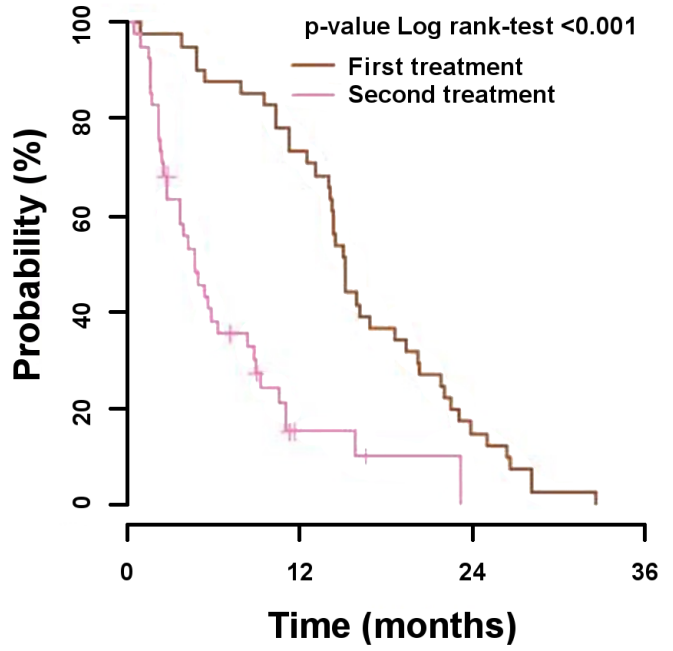

Figure 1. Progression free survival (PFS) for lenalidomide treatment $(\mathrm{N}=82$ treatment lines). Kaplan-Meier curves of PFS for the first and second lenalidomide treatment. The $\mathrm{Y}$ axis presents patients surviving without progression in percent and the $\mathrm{X}$ axis gives time of follow-up in months. The first lenalidomide treatment is in dark grey and the second is in light grey. P-value is by Log rank-test

Table 3. Progression free survival (PFS) for lenalidomide treatment.

\begin{tabular}{lcc}
\hline PFS & $\begin{array}{c}\text { First treatment with } \\
\text { lenalidomide } \\
(\mathrm{N}=41)\end{array}$ & $\begin{array}{c}\text { Second treatment } \\
\text { with lenalidomide } \\
(\mathrm{N}=41)\end{array}$ \\
\hline Median $(95 \% \mathrm{CI})$ & $15.2(14.2-16.2)$ & $4.8(3.0-6.6)$ \\
6 months $(95 \% \mathrm{CI})$ & $87.8(73.2-94.7)$ & $37.9(23.2-52.5)$ \\
12 months $(95 \% \mathrm{CI})$ & $73.2(56.8-84.2)$ & $15.1(5.8-28.6)$ \\
24 months $(95 \% \mathrm{CI})$ & $14.6(5.9-27.0)$ & - \\
\hline
\end{tabular}

exposure to bortezomib, thalidomide or carfilzomib before the second lenalidomide treatment was not established as a risk factor for repeated lenalidomide treatment success.

Moreover, the outcome of second lenalidomide treatment was independent of all the following; gender, age, ISS or DS stage, Eastern Cooperative Oncology Group (ECOG) performance status, type and amount of paraprotein or light chains, the levels of beta2microglobulin, albumin, lactate dehydro- 
Table 4. Subgroup PFS analysis.

\begin{tabular}{|c|c|c|}
\hline & $\begin{array}{c}\text { HR }(95 \% \mathrm{CI}) \text { for PFS } \\
\text { from second treatment } \\
\text { with lenalidomide }\end{array}$ & p-value \\
\hline \multicolumn{3}{|c|}{ Characteristics associated with the first treatment with lenalidomide } \\
\hline 2nd line of therapy & $0.852(0.302-2.399)$ & 0.761 \\
\hline 3rd line of therapy & $2.229(0.753-6.599)$ & 0.148 \\
\hline 4 th or higher line of therapy & $1.631(0.465-5.720)$ & 0.445 \\
\hline $\begin{array}{l}\text { Bortezomib in any previous } \\
\text { therapy }\end{array}$ & $2.016(0.273-14.896)$ & 0.492 \\
\hline $\begin{array}{l}\text { Thalidomide in any previous } \\
\text { therapy }\end{array}$ & $0.891(0.446-1.778)$ & 0.743 \\
\hline \multicolumn{3}{|c|}{ Characteristics at second treatment with lenalidomide } \\
\hline Age (years) (unit increase) & $0.950(0.905-0.998)$ & 0.043 \\
\hline Sex: women & reference & \\
\hline Sex: men & $1.129(0.800-1.592)$ & 0.490 \\
\hline ISS: stage 1 & reference & \\
\hline ISS: stage 2 & $1.889(0.801-4.453)$ & 0.146 \\
\hline ISS: stage 3 & $1.807(0.741-4.408)$ & 0.194 \\
\hline Durie-Salmon: stage I & reference & \\
\hline Durie-Salmon: stage II & $1.230(0.223-6.797)$ & 0.812 \\
\hline Durie-Salmon: stage III & $1.515(0.359-6.384)$ & 0.571 \\
\hline Durie-Salmon substage: A & reference & \\
\hline Durie-Salmon substage: B & $0.776(0.377-1.597)$ & 0.491 \\
\hline Performance status: 0 & reference & \\
\hline Performance status: 1 & $1.935(0.652-5.742)$ & 0.234 \\
\hline Performance status: $2-3$ & $1.849(0.593-5.769)$ & 0.290 \\
\hline M-protein type: $\operatorname{IgG}$ & reference & \\
\hline M-protein type: IgA & $0.943(0.381-2.334)$ & 0.898 \\
\hline M-protein type: LC only & $1.227(0.421-3.572)$ & 0.708 \\
\hline Nonsecretory & $0.209(0.028-1.577)$ & 0.129 \\
\hline Light chain type: kappa & reference & \\
\hline Light chain type: lambda & $1.810(0.884-3.707)$ & 0.105 \\
\hline Beta2 microglobulin (mg/l) & $1.092(0.954-1.251)$ & 0.203 \\
\hline Albumin level (g/l) & $1.007(0.949-1.069)$ & 0.809 \\
\hline LDH level & $1.129(0.943-1.353)$ & 0.188 \\
\hline Creatinine level (umol/l) & $1.004(0.993-1.015)$ & 0.450 \\
\hline Calcium total level $(\mathrm{mmol} / \mathrm{l})$ & $2.306(0.206-25.817)$ & 0.498 \\
\hline Hemoglobin level (g/l) & $0.990(0.968-1.012)$ & 0.379 \\
\hline Thrombocyte count (10E9/l) & $1.002(0.997-1.006)$ & 0.398 \\
\hline Serum M-protein level (g/l) & $1.001(0.984-1.019)$ & 0.889 \\
\hline $\begin{array}{l}\text { Bone marrow aspiration cytology: } \\
10-20 \%\end{array}$ & $0.227(0.010-5.369)$ & 0.358 \\
\hline $\begin{array}{l}\text { Bone marrow aspiration cytology: } \\
>20\end{array}$ & $0.376(0.017-8.084)$ & 0.532 \\
\hline Osteolytic lesions: more than 2 & $0.624(0.164-2.379)$ & 0.490 \\
\hline Extramedullary mass: yes & $1.415(0.536-3.731)$ & 0.483 \\
\hline
\end{tabular}

Univariate cox regression model for characteristics measured at second treatment with lenalidomide and PFS from this treatment line

genase, creatinine, calcium, hemoglobin, thrombocytes, infiltration of bone marrow, the number of osteolytic lesions or the presence of extramedullary disease. The dependence of treatment results on basic patients' characteristics before the second treatment is summarized in Table 4.
Table 5. Type of toxicity for the first and second treatment with lenalidomide.

\begin{tabular}{|c|c|c|c|}
\hline $\begin{array}{l}\text { Toxicity, n (\%) } \\
(\mathrm{N}=41)\end{array}$ & & $\begin{array}{c}\text { First treatment } \\
\text { with lenalidomide }\end{array}$ & $\begin{array}{l}\text { Second treatment } \\
\text { with lenalidomide }\end{array}$ \\
\hline \multirow[t]{3}{*}{ Anaemia } & grade 1 & $24(58.5 \%)$ & $18(43.9 \%)$ \\
\hline & grade 2 & $6(14.6 \%)$ & $12(29.3 \%)$ \\
\hline & grade 3 & $5(12.2 \%)$ & $6(14.6 \%)$ \\
\hline \multirow[t]{3}{*}{ Neutropenia } & grade 1 & $9(22.0 \%)$ & $7(17.1 \%)$ \\
\hline & grade 2 & $6(14.6 \%)$ & $12(29.3 \%)$ \\
\hline & grade 3 & $24(58.5 \%)$ & $10(24.4 \%)$ \\
\hline \multirow[t]{3}{*}{ Thrombocytopenia } & grade 1 & $20(48.8 \%)$ & $17(41.5 \%)$ \\
\hline & grade 2 & $5(12.2 \%)$ & $2(4.9 \%)$ \\
\hline & grade 3 & $8(19.5 \%)$ & $8(19.5 \%)$ \\
\hline \multirow[t]{2}{*}{ Infection } & grade 1 & $2(4.9 \%)$ & $2(4.9 \%)$ \\
\hline & grade 2 & $14(34.1 \%)$ & $14(34.1 \%)$ \\
\hline \multirow[t]{2}{*}{ Fatigue } & grade 1 & $14(34.1 \%)$ & $9(22.0 \%)$ \\
\hline & grade 3 & $5(12.2 \%)$ & $10(24.4 \%)$ \\
\hline \multirow[t]{3}{*}{ Neuropathy } & grade 1 & $13(31.7 \%)$ & $7(17.1 \%)$ \\
\hline & grade 2 & $5(12.2 \%)$ & $5(12.2 \%)$ \\
\hline & grade 3 & $2(4.9 \%)$ & $0(0.0 \%)$ \\
\hline \multirow[t]{3}{*}{ Diarrhoea } & grade 1 & $1(2.4 \%)$ & $1(2.4 \%)$ \\
\hline & grade 2 & $4(9.8 \%)$ & $4(9.8 \%)$ \\
\hline & grade 3 & $1(2.4 \%)$ & $2(4.9 \%)$ \\
\hline \multirow[t]{2}{*}{ Constipation } & grade 1 & $3(7.3 \%)$ & $1(2.4 \%)$ \\
\hline & grade 2 & $6(14.6 \%)$ & $0(0.0 \%)$ \\
\hline \multirow[t]{3}{*}{ Nausea } & grade 1 & $1(2.4 \%)$ & $2(4.9 \%)$ \\
\hline & grade 2 & $3(7.3 \%)$ & $2(4.9 \%)$ \\
\hline & grade 3 & $0(0.0 \%)$ & $1(2.4 \%)$ \\
\hline \multirow[t]{3}{*}{ Anorexia } & grade 1 & $2(4.9 \%)$ & $0(0.0 \%)$ \\
\hline & grade 2 & $3(7.3 \%)$ & $1(2.4 \%)$ \\
\hline & grade 3 & $1(2.4 \%)$ & $0(0.0 \%)$ \\
\hline
\end{tabular}

Count (relative frequencies)

Adverse events. As expected, treatment toxicity was mainly haematologic. In the first lenalidomide treatment, the most common side effect was anemia - 73\% (30/41), but at grades 1-2 this was not severe. Other less serious side effects of treatment were thrombocytopenia - $60.9 \%(25 / 41)$, infections $-39.0 \%(16 / 41)$ and neutropenia $-36.5 \%(15 / 41)$. The most common severe (grade 3 ) side effects of treatment were neutropenia $-58.5 \%(24 / 41)$ and infections - 22\% (9/22).

In the second lenalidomide treatment, the most frequent side effect was also anemia $-73 \%$ (30/41). Other less serious adverse effects (grades 1-2) were thrombocytopenia - 46\% (19/41), neutropenia - 46\% (19/41) and infections - 39\% (16/41); and the most common severe (grade 3) adverse effects of this treatment were neutropenia - 24.4\%, 10/41) and thrombocytopenia $-19.5 \%(8 / 41)$. No grade 4 toxicity was observed during first or second lenalidomide treatments and no patients died due to the treatment: - results are summarized in Table 5. 
Table 6. Comparison between lenalidomide retreatment, daratumumab monotherapy and pomalidomide dexamethasone.

\begin{tabular}{|c|c|c|c|}
\hline & $\begin{array}{c}\text { Lenalidomide } \\
\text { retreatment }\end{array}$ & $\begin{array}{c}\text { Daratumumab } \\
\text { monotherapy [19] }\end{array}$ & $\begin{array}{l}\text { Pomalidomide-dexamethasone } \\
{[17]}\end{array}$ \\
\hline Number of patients & 41 & 106 & 302 \\
\hline Median previous treatment lines (range) & $3(2-6)$ & $5(2-14)$ & $5(2-14)$ \\
\hline Previous bortezomib treatment & $95.1 \%$ & $99 \%$ & $100.0 \%$ \\
\hline Previous lenalidomide treatment & $100.0 \%$ & $100.0 \%$ & $100.0 \%$ \\
\hline Previous PBSCT & $48.7 \%$ & $100.0 \%$ & $71.0 \%$ \\
\hline Refractory to the last treatment line & $100.0 \%$ & $97.0 \%$ & $82.0 \%$ \\
\hline PFS median (months) & 4.8 & 3.7 & 4.0 \\
\hline OS median (months) & 11.9 & 17.5 & 12.7 \\
\hline
\end{tabular}

\section{Discussion}

Advances in MM treatment have significantly extended patient survival [15-19], the number of pretreated and refractory patients has increased [3]. Despite expanding treatment options, in real-life clinical practice we are forced to choose between repeated cancer therapy and palliative treatment. Lenalidomide is an effective antimyeloma drug standardly used to treat relapsed $\mathrm{MM}$ in the Czech Republic. Combined therapy with lenalidomide and dexamethasone achieved median PFS of 25.5 months in newly diagnosed MM patients [9] and 17.6 months in relapsed MM patients [18]. Lenalidomide retreatment has previously been described in only one study [11]. Therein, 48 patients were retreated with lenalidomide after first-line lenalidomide treatment. The median of treatment lines before the second treatment was 2; lenalidomide was used in newly-diagnosed disease in $42 \%$ of patients and only $24 \%$ had prior bortezomib treatment before second-line lenalidomide treatment. That study therefore had a considerably less pretreated group of patients than ours, and their median time of lenalidomide treatment was 7 months compared to 4 months for our group. This was most likely due to Czech health insurance rules which forced us to treat patients with only a limited dose of lenalidomide and to discontinue treatment in those who had not achieved partial response (PR) after 4 cycles. In second lenalidomide treatment, $54 \%$ of patients achieved PR and better response. The median PFS for the second lenalidomide treatment was 16.0 months. The ORR was only $25 \%$ in the subgroup that progressed after the first lenalidomide treatment, so PFS and other treatment results were not determined. [11].

Our patient group was significantly more pretreated than in the quoted study and lenalidomide retreatment was the last-available treatment option for all our patients at the time. Only four lenalidomide cycles in case of insufficient response after four cycles and maximum cumulative dose of $4200 \mathrm{mg}$ of lenalidomide were reimbursed. It is clear that these limitations had a significant impact on both our treatment length and results. We suggest that if prolonged administration of lenalidomide was reimbursed, therapeutic response could occur later, and we could achieve better treatment results. We also consider that the limitation of the cumulative dose of lenalidomide affected the number of patients who progressed after treatment withdrawal due to this rule. A maximum of eleven cycles in the second line was reached for the same reason. Hence, PFS and OS are clearly influenced by the length of lenalidomide dosing $[9,20]$.

Pomalidomide or daratumumab are current possible treatment choices for refractory and heavily pre-treated MM patients. In a randomized trial evaluating combined treatment with pomalidomide and dexamethasone [17], there was a similarly pre-treated group of patients to our group. All these had received previous bortezomib and lenalidomide treatment, $57 \%$ had received thalidomide and $71 \%$ underwent high-dose chemotherapy with autologous transplantation. Results highlighted that $82 \%$ of these patients were refractory to the last treatment, and the number of previous lines of therapy was 5 . In another trial evaluating daratumumab monotherapy, $99 \%$ of patients were pre-treated with bortezomib, $100 \%$ with lenalidomide and $44 \%$ with thalidomide [19]. Further, $80 \%$ underwent high dose chemotherapy followed by autologous stem-cell transplant. The median of prior treatment lines was 5 , and $97 \%$ of patients were refractory to the last treatment line. It should be noted here that $63 \%$ of patients in this group were pretreated with carfilzomib and $50 \%$ with pomalidomide. Although our group differed in the number of previous therapy lines, our patients had similar resistance to previous treatment types, and all were refractory to the last administered therapy.

In a clinical trial with pomalidomide and dexamethasone [17], median PFS was 4.0 months and median OS was 12.7 months. Meanwhile, daratumumab monotherapy [19] achieved a median PFS 3.7 months and median OS of 17.5 months. These results are listed in Table 6. In those trials, both pomalidomide and daratumumab were administered until disease progression. Although treatment duration in our patients was limited by Czech health insurance rules, our analytic results are comparable with those from both the pomalidomide and daratumumab treatments $[17,19]$. Moreover, our literature survey and this paper establish that 
the direct comparison of repeated lenalidomide treatment with pomalidomide or daratumumab in a large randomized trial is obviously lacking. A surprising finding from our analysis is that PFS length in the second lenalidomide treatment is independent of response to its first treatment. This is explained by MM clonal theory [21]. We assume that even if the first lenalidomide treatment did not achieve treatment response, the second treatment can affect other plasma cell clones selected by previous non-lenalidomide therapy. The results of the second lenalidomide treatment did not depend on age or ECOG performance status. This might encourage use of repeated lenalidomide treatment in elderly and frail patients who can benefit from home-administered oral treatment. Toxicity of lenalidomide retreatment was tolerable and usually moderate at grade 1-2, and its incidence was comparable between the two treatment lines without clinical significance.

Results of our analysis demonstrate the effectiveness and usefulness of repeated lenalidomide treatment in a significantly pre-treated group of relapsed and refractory $\mathrm{MM}$ patients. In a broad spectrum of patients, repeated lenalidomide treatment is a comparable alternative to pomalidomide or daratumumab treatment, and its great advantage is its much lower cost.

Acknowledgments: The authors thank all patients and their caregivers for participating in this study, and also John B. Smith for proofreading the manuscript. This work was supported by The Czech Ministry of Health Grant No.AZV 15-29508A and specific research grant - MUNI/A/1106/2016

\section{References}

[1] RAJKUMAR SV, KUMAR S. Multiple Myeloma: Diagnosis and Treatment. Mayo Clin Proc 2016; 91: 101-119. https:// doi.org/10.1016/j.mayocp.2015.11.007

[2] SAN-MIGUEL JF, MATEOS MV. Can multiple myeloma become a curable disease? Haematologica 2011; 96: 12461248. https://doi.org/10.3324/haematol.2011.051169

[3] KUMAR SK, LEE JH, LAHUERTA JJ, MORGAN G, RICHARDSON PG et al. Risk of progression and survival in multiple myeloma relapsing after therapy with IMiDs and bortezomib: a multicenter international myeloma working group study. Leukemia 2012; 26: 149-157. https://doi.org/10.1038/ leu.2011.196

[4] Sonneveld P, Broijl A. Treatment of relapsed and refractory multiple myeloma. Haematologica 2016; 101: 396-406. https://doi.org/10.3324/haematol.2015.129189

[5] SONNEVELD P, GOLDSCHMIDT H, ROSINOL L, BLADÉ J, LAHUERTA JJ et al. Bortezomib-based versus Nobortezomib - based induction treatment before autologous stemcell transplantation in patients with previously untreated multiple myeloma: a meta-analysis of phase III randomized, controlled trials. J Clin Oncol 2013; 31: 3279-3287. https:// doi.org/10.1200/JCO.2012.48.4626
[6] MOREAU P, HULIN C, MACRO M, CAILLOT D, CHALETEIX C et al. VTD is superior to VCD prior to intensive therapy in multiple myeloma: results of the prospective IFM2013-04 trial. Blood 2016; 127: 2569-2574. https://doi. org/10.1182/blood-2016-01-693580

[7] DIMOPOULOS MA, CHEN C, SPENCER A, NIESVIZKY $\mathrm{R}$, ATTAL $\mathrm{M}$ et al. Long-term follow-up on overall survival from the MM-009 and MM-010 phase III trials of lenalidomide plus dexamethasone in patients with relapsed or refractory multiple myeloma. Leukemia 2009; 23: 2147-2152. https://doi.org/10.1038/leu.2009.147

[8] STADTMAUER EA, WEBER DM, NIESVIZKY R, BELCH $\mathrm{A}, \mathrm{PRINCE} \mathrm{MH}$ et al. Lenalidomide in combination with dexamethasone at first relapse in comparison with its use as later salvage therapy in relapsed or refractory multiple myeloma. Eur J Haematol 2009; 82: 426-432. https://doi. org/10.1111/j.1600-0609.2009.01257.x

[9] BENBOUBKER L, DIMOPOULOS MA, DISPENZIERI A, CATALANO J, BELCH AR et al. Lenalidomide and dexamethasone in transplant-ineligible patients with myeloma. N Engl J Med 2014; 371: 906-917. https://doi.org/10.1056/ NEJMoa1402551

[10] DURIE BG, HOERING A, ABIDI MH, RAJKUMAR SV, EPSTEIN $J$ et al. Bortezomib with lenalidomide and dexamethasone versus lenalidomide and dexamethasone alone in patients with newly diagnosed myeloma without intent for immediate autologous stem-cell transplant (SWOG S0777): a randomised, open-label, phase 3 trial. Lancet 2017; 389: 519-52. https://doi.org/10.1016/S0140-6736(16)31594-X

[11] MADAN S, LACY MQ, DISPENZIERI A, GERTZ MA, BUADI $F$ et al. Efficacy of retreatment with immunomodulatory drugs (IMiDs) in patients receiving IMiDs for initial therapy of newly diagnosed multiple myeloma. Blood 2011; 118:1763-1765. https://doi.org/10.1182/ blood-2011-04-350009

[12] RAJKUMAR SV, DIMOPOULOS MA, PALUMBO A, BLADE J, MERLINI G et al. International Myeloma Working Group updated criteria for the diagnosis of multiple myeloma. Lancet Oncol 2014;15: e538-5e48. https://doi. org/10.1016/S1470-2045(14)70442-5

[13] PALUMBO A, ANDERSON K. Multiple myeloma. N Engl J Med. 2011; 364: 1046-1060. https://doi.org/10.1056/NEJMra1011442

[14] LAROCCA A, CAVALLO F, BRINGHEN S, DI RAIMONDO F, FALANGA A et al. Aspirin or enoxaparin thromboprophylaxis for patients with newly diagnosed multiple myeloma treated with lenalidomide. Blood 2012; 119: 933-939. https://doi.org/10.1182/blood-2011-03-344333

[15] RAJKUMAR SV. Myeloma Today: Disease Definitions and Treatment Advances. Am J Hematol 2016; 91: 90-100. https://doi.org/10.1002/ajh.24236

[16] CORNELL RF, KASSIM AA. Evolving paradigms in the treatment of relapsed/refractory multiple myeloma: increased options and increased complexity. Bone Marrow Transplant 2016; 51: 479-491. https://doi.org/10.1038/ bmt.2015.307 
[17] SAN MIGUEL JF, WEISEL K, MOREAU P, LACY M, SONG $\mathrm{K}$ et al. Pomalidomide plus low-dose dexamethasone versus high-dose dexamethasone alone for patients with relapsed and refractory multiple myeloma (MM-003): a randomised, open-label, phase 3 trial. Lancet Oncol 2013; 14: 1055-1066. https://doi.org/10.1016/S1470-2045(13)70380-2

[18] STEWART AK, RAJKUMAR SV, DIMOPOULOS MA, MASSZI T, SPICKA I et al. Carfilzomib, lenalidomide, and dexamethasone for relapsed multiple myeloma. N Engl J Med 2015; 372: 142-152. https://doi.org/10.1056/NEJMoa1411321
[19] LONIAL S, WEISS BM, USMANI SZ, SINGHAL S, CHARI A et al. Daratumumab monotherapy in patients with treatment-refractory multiple myeloma (SIRIUS): an open-label, randomised, phase 2 trial. The Lancet 2016; 387; 1551-1560. https://doi.org/10.1016/S0140-6736(15)01120-4

[20] PALUMBO A, HAJEK R, DELFORGE M, KROPFF M, PETRUCCI MT et al. Continuous lenalidomide treatment for newly diagnosed multiple myeloma. N Engl J Med 2012; 366 : 1759-1769. https://doi.org/10.1056/NEJMoa1112704

[21] KEATS JJ, CHESI M, EGAN JB, GARBITT VM, PALMER $\mathrm{SE}$ et al. Clonal competition with alternating dominance in multiple myeloma. Blood 2012; 120: 1067-1076. https://doi. org/10.1182/blood-2012-01-405985 